\title{
Quantitative proteomic characterization of human sperm cryopreservation: using data-independent acquisition mass spectrometry
}

\author{
Longlong Fu $u^{1,2}$, Qi An ${ }^{1,2,3}$, Kaishu Zhang ${ }^{4}$, Ying Liu ${ }^{5}$, Yue Tong ${ }^{1,2,3}$, Jianfeng Xu ${ }^{1,2}$, Fang Zhou ${ }^{1,2}$, Xiaowei Wang ${ }^{1,2}$, \\ Ying Guo ${ }^{1,2}$, Wenhong Lu ${ }^{1,3^{*}}$, Xiaowei Liang ${ }^{2}$ and Yiqun Gu ${ }^{1,2,3^{*}}$
}

\begin{abstract}
Background: Human sperm cryopreservation is a simple and effective approach for male fertility preservation. Methods: To identify potential proteomic changes in this process, data-independent acquisition (DIA), a technology with high quantitative accuracy and highly reproducible proteomics, was used to quantitatively characterize the proteomics of human sperm cryopreservation.

Results: A total of 174 significantly differential proteins were identified between fresh and cryoperservated sperm: 98 proteins decreased and 76 proteins increased in the cryopreservation group. Bioinformatic analysis revealed that metabolic pathways play an important role in cryopreservation, including: propanoate metabolism, glyoxylate and dicarboxylate metabolism, glycolysis/gluconeogenesis, and pyruvate metabolism. Four different proteins involved in glycolysis were identified by Western blotting: GPI, LDHB, ADH5, and PGAM1.
\end{abstract}

Conclusions: Our work will provide valuable information for future investigations and pathological studies involving sperm cryopreservation.

Keywords: Cryopreservation, Fertility preservation, Reproductive techniques, assisted, Metabolic networks and pathways

\section{Background}

Human sperm cryopreservation plays an important role in the treatment of male infertility when the male partner has severe abnormalities in semen parameters [1]. Furthermore, this technique is a simple and effective approach to male fertility preservation $[2,3]$, and is the only approach used in the clinic in many countries [4]. However, the cryopreservation protocols will induce a notable decrease in sperm motility, as well as affecting other parameters, including: membrane and acrosome integrity, DNA fragment, and reactive oxygen species [5, 6]. Certain intracellular and extracellular factors have been shown to lead to cryo-damage $[7,8]$. However, the

\footnotetext{
*Correspondence: wenhonglu16@163.com; guyq16@163.com

${ }^{1}$ National Health Commission Key Laboratory of Male Reproductive Health,

National Research Institute for Family Planning, Beijing 100081, China

Full list of author information is available at the end of the article
}

related pathogenesis of sperm cryo-damage during the process of cryopreservation shaould be clarified by further study.

Proteomics, especially sperm proteomics, is a new field in human reproduction studies. Ptoteomics can elucidate complex biological systems, including sperm motility and fertilization, and it can discover the potential pathogenic mechanisms and the biomarkers associated with male infertility $[9,10]$. Data-independent acquisition (DIA) is, a novel proteomics technology, based on acquiring fragment ion information for all precursor ions within a certain range of $\mathrm{m} / \mathrm{z}$ values [11]. Different from datadependent acquisition (DDA), the DIA strategy has the characteristics of high quantitative accuracy and high reproducibility; and the experimental method has broad applicability: up to 5000 proteins can be detected and quantified in one experiment [11, 12]. To the best of our

(c) The Author(s). 2019 Open Access This article is distributed under the terms of the Creative Commons Attribution 4.0 International License (http://creativecommons.org/licenses/by/4.0/), which permits unrestricted use, distribution, and 
knowledge, this technique has not previously been applied to the study of human cryopreserved sperm.

For these reasons, the aim of the current study is to compare the proteomic differences between fresh and cryopreserved human sperm, using DIA mass data. A further understanding the sperm proteins would be very helpful in explaining sperm cryoinjury and may establish biomarkers for sperm motility.

\section{Methods}

\section{Ethics statement and sample collection}

The study was approved by National Research Institute for Family Planning Ethics Committee on Human Subjects (2018018). Ejaculates were obtained from healthy semen donors attending the Human Sperm Bank, National Research Institute for Family Planning, in Beijing China. Informed consent was obtained from all the sperm donors involved in the study.

\section{Study design and sample collection}

Paired design was applied to this study. Every semen sample was divided into two parts: one for the cryopreserved group and the other for the fresh group. Our focus was the difference between the cryopreserved and fresh human sperm.

A total of 14 semen samples from 14 qualified healthy sperm donors were collected. The ages of the sperm donors were between 22 years and 29 years. One ejaculate was collected from these volunteers by masturbation after 3-5 days of sexual abstinence. Routine semen analyses were performed by Computer Aided Sperm Analysis (CASA)(HTM-IVOS, USA), according to the World Health Organization (WHO 2010) guidelines.

Among the whole sample of each group, 9 cryopreserved and fresh samples were used for proteomics analysis. Similar to the previous study [13], the 9 donors' ejaculates (each one divided into fresh and cryopreserved vials) were divided into 3 groups of 3 . Then 5 for Western blot analysis.

\section{Semen cryopreservation protocols}

After complete liquefaction, $2 \mathrm{~mL}$ of each semen sample was divided into two parts: one for cryopreservation and the other placed in a $37^{\circ} \mathrm{C}$ water bath as the control. Glycerol-egg-yolk-citrate (GEYC) was used as cryoprotectant containing $15 \%$ glycerol, $20 \%$ egg yolk, $1.3 \%$ glycine, $1.5 \%$ glucose, and $1.3 \%$ sodium citrate tribasic dehydrate, with a pH of 6.8-7.2. One volume of GEYC was added to two volumes of semen, drop by drop with swirling, and the mixture was incubated at $30-35^{\circ} \mathrm{C}$ for 5 min. A slow sperm freezing method was performed according to the standardized programmable freezers (Kryo 360-1.7, Planner, United Kingdom) in our unit $[12,14]$. Briefly, one volume of GEYC cryoprotectant was added to two volumes of semen, and the program was as follows: the samples tubes waerw cooled at $1.5^{\circ} \mathrm{C}$ per minute from $20^{\circ} \mathrm{C}$ to $-6^{\circ} \mathrm{C}$, at $6^{\circ} \mathrm{C}$ per minute to $100{ }^{\circ} \mathrm{C}$, and at $-100^{\circ} \mathrm{C}$ for $30 \mathrm{~min}$, then the sample tubes were transferred to liquid nitrogen. After being preserved in the liquid nitrogen for a minimum of 2 days, a small portion $(10 \mu \mathrm{L})$ of the frozen sample was thawed for sperm quality assessment.

\section{Protein extraction and digestion}

Protein extraction and digestion were performed as described previously [15]. The fresh and cryopreserved semen were centrifuged at $800 \mathrm{~g}$ for $10 \mathrm{~min}$ to remove seminal plasma, round cells and the cryoprotectant. Then, the sperm were washed three times with phosphatebuffered saline (PBS). Five hundred microliters of lysis buffer [8 M UREA, $100 \mathrm{mM}$ Tris-HCl, pH 7.6, $1 \mathrm{mM}$ PMSF (phenylmethylsulfonyl fluoride)(Roche, Germany)] was added to each sample. Then, the samples were sonicated(Sonics, USA) at 20 joules for $2 \mathrm{~s} \times 10$ at intervals of 15 $\mathrm{s}$, and centrifuged at $18000 \mathrm{~g}$ for $15 \mathrm{~min}$, after which the supernatant was extracted. Quantification was performed using the BCA method. The protein sample was frozen at $-80^{\circ} \mathrm{C}$. An aliquot of $20 \mu \mathrm{g}$ was used for each sample to build the mixed and pooled library. Six samples and the pooled samples were subjected to enzymatic hydrolysis using a FASP (filter-aided sample preparation) enzymatic method.

Then, protein digestion was performed using the FASP protocol. In brief, protein $(200 \mu \mathrm{g})$ was diluted with 50 $\mathrm{mM}$ dithiothreitol and incubated for $40 \mathrm{~min}$ at $56^{\circ} \mathrm{C}$. Further operation was carried out using the ultrafiltration tube method. The ultrafiltration tube was placed in a collection tube, and the protein sample was added to the ultrafiltration tube and centrifuged at $12000 \mathrm{~g}$ for 15 min. One hundred microliters of urea buffer containing $50 \mathrm{mM}$ iodoacetamide was added to the ultrafiltration tube and it was then incubated for $20 \mathrm{~min}$ in the dark. The sample was washed twice by adding $100 \mu \mathrm{L}$ urea buffer and centrifugede at $12000 \mathrm{~g}$ for $10 \mathrm{~min}$ to remove irrelevant substances. Then, $80 \mu \mathrm{L}$ of $50 \mathrm{mM}$ trypsin in $\mathrm{NH}_{4} \mathrm{HCO}_{3}$ was added to ultrafiltration tube, and the protein-to-enzyme ratio was 50:1. The samples were incubated at $37^{\circ} \mathrm{C}$ for $16 \mathrm{~h}$, and the released peptides were collected through centrifugation and directly determined by the protein concentration detection mode in the Nano-Drop instrument.

\section{Spectral library generation}

The samples $(1 \mu \mathrm{g})$ were analyzed on an EASY-Nano-LC mass spectrometer (Thermo, USA). The peptides were separated using $0.1 \%$ formic acid, containing iRT standard peptide(Buffer A) and acetonitrile containing 0.1\% formic acid(Buffer B), along a linear gradient from 3 to 
$32 \%$ at $300 \mathrm{~nL} / \mathrm{min}$ for $120 \mathrm{~min}$. The gradient of chromatographic separation was as follows: $3-7 \%$ buffer B for 0-3 min, 7-20\% buffer B for $80 \mathrm{~min}, 20-32 \%$ buffer B for $24 \mathrm{~min}, 32-90 \%$ buffer B for $1 \mathrm{~min}$, and $100 \%$ buffer B for $120 \mathrm{~min}$. For DDA, the source was operated by Orbitrap Fusion (Thermo Scientific, USA), at $2.1 \mathrm{kV}$. The DDA scheme included a full MS survey scan from $\mathrm{m} /$ z350 to $\mathrm{m} / \mathrm{z} 1500$ at a resolution of $60 \mathrm{k}$ full-width halfmaximum (FWHM)(at $\mathrm{m} / \mathrm{z} 200)$ with automatic gain control(AGC) set to 4E5 (maximum injection time of 50 $\mathrm{ms}$ ). The parameters of MS2 were as follows: $30 \mathrm{k}$ FWHM (@ $\mathrm{m} / \mathrm{z} 200$ ), isolation window $1.6 \mathrm{Th}$, AGC set to $4 \mathrm{e} 5$ (maximum injection time of $50 \mathrm{~ms}$ ). For, high-energy collision dissociation (HCD): MS2 Activation (collision energy: 35 ) was used, and dynamic exclusion was set to 40s.

For the generation of the spectral library, DDA data analysis was performed using Protein Discoverer 2.1 SP1(SEQUEST HT). The database is human proteins database from UniProt: "uniprot-organism-9606 + reviewed-yes.fasta, " and iRT peptide sequences were added to the database: (>Biognosys|iRT-Kit|Sequence_fusion LGGNEQVTRYILA GVENSKGTFIDPGGVIRGTFIIDPAAVIRGAGSSEPVTG LDAKTPVISGGPYEYRVEATFGVDESNAKTPVITGAPYE YRDGLDAASYYAPVRADVTPADFSEWKLFLQFGAQGS SPFLK).

Raw data were analyzed according to the user guide of the software. The parameters were set as follows: the initial mass tolerance for precursor ions was: $10 \mathrm{ppm}$, and the mass tolerance for product ion spectra was $0.02 \mathrm{Da}$. Tryptic cleavage was selected, and the maximum allowable number of missing cleavage was 2. Carbamidomethyl of cysteine was set as the fixed modification, while oxidation of methionine and $\mathrm{N}$ terminal acetylation were set as variable modifiers. The identifications were filtered to satisfy an FDR of $1 \%$, and the unique peptide number per protein was greater than one. Then, the result was used to build the DDA spectral library in Spectronaut Pulsar $\mathrm{X}$ (Biognosys, Switzerland). The library parameters used the default optimal parameter "GBS factory setting".

\section{Protein identification and quantitation}

For DIA, $2 \mu$ g peptides was taken from each sample and mixed with appropriate iRT standard peptides. Each sample was tested by DIA mass spectrometry for $2 \mathrm{~h}$. The method consisted of a full MS1 scan at a resolution of $60 \mathrm{~K}$ from m/z 350 to m/z 1500, with AGC set to 4E5 (maximum injection time of $50 \mathrm{~ms}$ ), followed by 46 DIA windows acquired at a resolution of $30 \mathrm{~K} \mathrm{FWHM} \mathrm{with}$ AGC set to $5 \mathrm{e} 5$ (maximum injection time of $55 \mathrm{~ms}$ ); HCD: MS2 activation (collision energy: 35 ).

Finally, qualitative and quantitative analyses of DIA raw data were performed in Spectronaut Pulsar X. Database parameters were as follows: peptides FDR $\backslash$ PSM FDR $\backslash$ proteins FDR was 1\%; at least three proteins were selected for each peptide, at most 6 optimal ion generation library spectra were selected, and iRT calibration $R^{2}>0.8$. The quantitative parameters were set as follows: the iTR standard used a nonlinear fit (local (non-linear) regression), the protein identification used a precursor Qvalue cutoff 0.01 , the protein Qvalue cutoff of 0.01 , the protein quantification used the peak area of sub-ions, and the average Intensity of at least three sub-ions was selected to quantify the protein. Protein quantification uses the ion peak area, and at least the average intensity quantification of three sub-ions is selected. Paired t-test was used as the analytical approach and $P$-value of 0.05 was set.

\section{Bioinformatics analysis}

All significantly differential proteins were used as input. The OmicsBean analysis tool [16] was used to retrieve the Gene Ontology Consortium categories, including: molecular function (MF), cellular component (CC) and biological process (BP). KEGG [17] pathway enrichment analysis was performed using the Kanehisa databases web service (https://www.kanehisa.jp/en/archive.html). The protein-protein interaction network (PPI) was constructed using the STRING [18] web service (http:// www.string-db.org/).

\section{Western blotting}

According to our previous experience [19], the sperm protein samples were separated by $10 \%$ SDS polyacrylamide gel electrophoresis. The samples were then transferred to a polyvinylidene fluoride membranes, blocked with $2 \%(\mathrm{w} / \mathrm{v})$ skim milk for $1 \mathrm{~h}$, and incubated overnight with the primary antibody of glucose-6-phosphate isomerase (GPI), lactate dehydrogenase B (LDHB), alcohol dehydrogenase 5 (ADH5), and phosphoglycerate mutase 1 (PGAM1) (1:1000) (Abclone, China) at $4{ }^{\circ} \mathrm{C}$ temperature. After three washes with TBST, the membranes were incubated with horseradish peroxidase (HRP) in combination with anti-IgG for $1 \mathrm{~h}$ at room temperature $\left(18-22^{\circ} \mathrm{C}\right)$. Enhanced chemiluminescence revealed immunoreactivity. The relative signal intensity of protein bands was analyzed with Quantity One v.4.6.2.

\section{Statistical analysis}

Data are expressed as the mean \pm SD and were analyzed with SPSS22.0 software (IBM, USA). Paired t-test was used as the analytical approach, and a $P$-value of 0.05 was set.

\section{Results}

\section{Sperm motility}

We aimed to assess the impact of cryopreservation on sperm motility (Table 1). Compared with fresh semen, a significant decrease in the percentage of progressive 
Table 1 Characteristics of motility of fresh and post-thaw human sperm $(n=14)($ Mean \pm SD)

\begin{tabular}{lllll}
\hline & Percentage of PR(\%) & VAP $(\mu \mathrm{m} / \mathrm{s})$ & VSL $(\mu \mathrm{m} / \mathrm{s})$ & $\mathrm{VCL}(\mu \mathrm{m} / \mathrm{s})$ \\
\hline Fresh sperm & $67.0 \pm 7.5$ & $42.4 \pm 0.9$ & $31.0 \pm 1.7$ & $65.2 \pm 1.2$ \\
Post-thaw sperm & $45.3 \pm 2.51^{*}$ & $37.8 \pm 2.4^{*}$ & $29 \pm 0.5^{*}$ & $58.6 \pm 5.8^{*}$ \\
\hline
\end{tabular}

*: $P<0.05$ compared with the Fresh group

$P R$ progressive; VAP average path velocity; VSL straight line velocity; $V C L$ curvilinear velocity.

sperm, and sperm motion parameters was observed in cryoperserved sperm, including average path velocity (VAP), straight line velocity (VSL), and curvilinear velocity (VCL).

\section{Quantitative results of differential proteins}

Compared with the spectral library, a total of 29,495 unique peptides and 5246 proteins were identified. Finally, 3790 proteins were quantitatively analyzed. Using the $1.5-$ fold or 0.67 -fold change and the FDR-adjusted $p$-value of 0.05 as cutoffs, 174 (4.6\%) significantly differential proteins were identified between fresh and cryopreserved sperm: 76 were increased and 98 were decreased in the cryopreserved group. The detail of the differential proteins due to cryopreservtaion are provided in the Additional file 1: Table S1.

\section{Gene ontology enrichment}

Enrichment analysis was performed based on the 174 significantly deregulated proteins. A total of 1544 biological processes(BPs), 275 cell components(CCs) and 261 molecular functions(MFs) were involved in the GO enrichment, and there were significant differences between the fresh and cryopreserved $\operatorname{sperm}(P<0.05)$. The number of differential proteins contained in each entry and its percentage of total differential protein are shown in Fig. 1. The detailed results of the GO enrichment are provided in Additional file 2: Table S2.

\section{KEGG pathway and protein-protein interaction (PPI) network analysis}

Pathway enrichment analysis was also performed by KEGG enrichment in order to identify the major biochemical pathways and signal transduction pathways. Several pathways were significantly disturbed in cryopreserved sperm, such as: ribosome, carbon metabolism and lysosome (Fig. 2). The details of the 16 pathways with significant differences are shown in Table 2. To show the main interactions and regulatory relationships of these proteins, the PPI network was constructed (Fig. 3).

According to the KEGG analysis, the results showed found that the metabolic pathways play an important role in cryopreservation (Fig. 2), including: propanoate metabolism, glyoxylate and dicarboxylate metabolism, glycolysi/gluconeogenesis, and pyruvate metabolism. Most of these pathways were down regulated in the cryopreserved sperm group (the green in the Fig. 3).

\section{Validation of the glycolysis metabolic proteins}

To further validate the outcome of the KEGG analysis, we used Western blotting to quantify the four dysregulated protein enzymes in glycolysis: GPI, LDHB, ADH5, and PGAM1.

These protein analysis results confirmed the previous genomic analysis of metabolomics, and the results confirmed the differential protein levels observed via $2 \mathrm{DE}$ (Fig. 4). The cryopreserved group had lower levels of

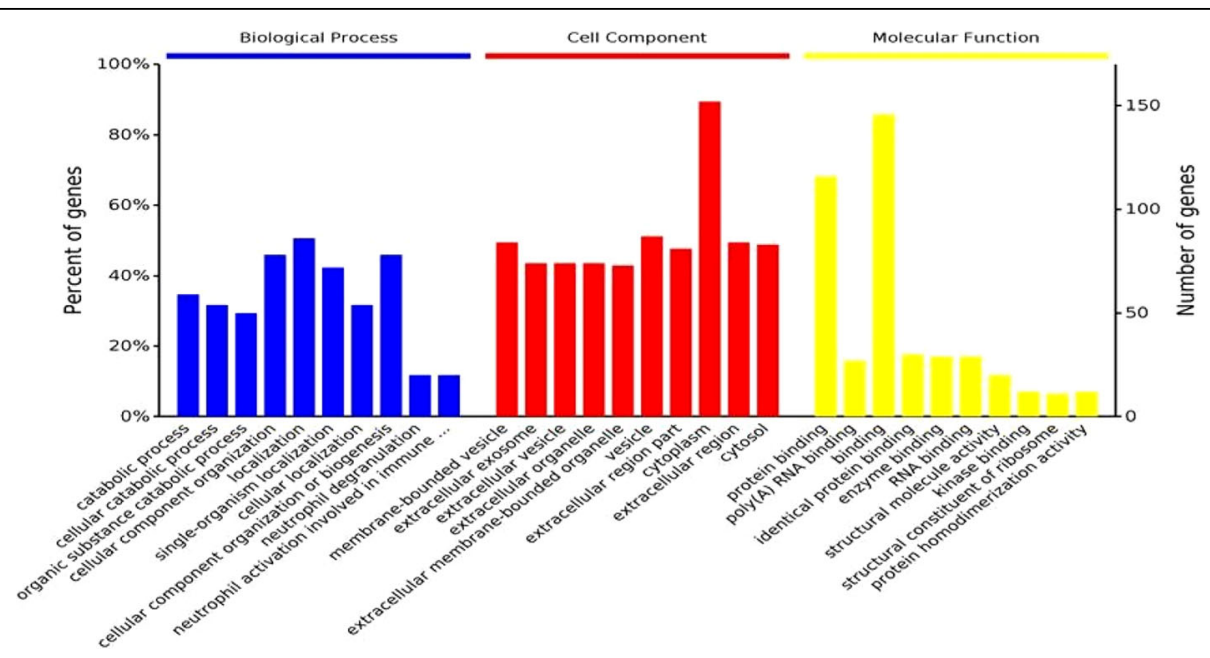

Fig. 1 Gene Ontology analysis classification and the enrichment result of differentially expressed proteins in the human freeze-thaw samples compared to the fresh group, in terms of Biological Process, Cell Component and Molecular Function 


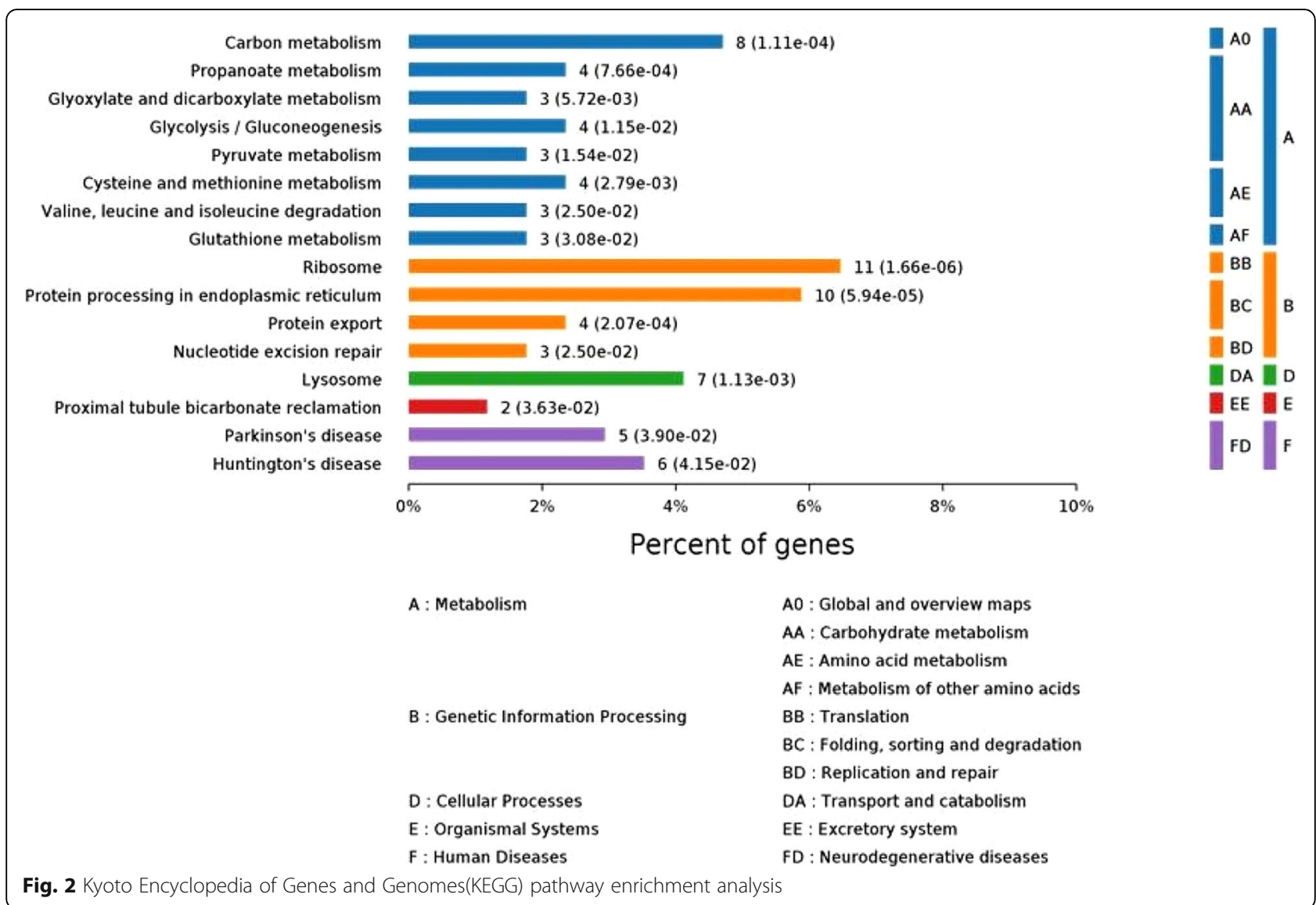

GPI, LDHB, and PGAM1. and a higher level of ADH5 than the fresh sperm (Fig. 4).

\section{Discussion}

Worldwide, the percentage of male infertility ranges between 20 and 70\% [20], and men with azoospermia or severe oligozoospermia will benefit from sperm cryopreservation. Furthermore, cryopreservation is a simple and effective technique for preserving fertility potential $[4,21]$. However, after sperm cryopreservation, too many sperm lose their motility and fertility [5, 22]. Some sperm proteins have been recognized to be associated with sperm quality, and the loss of these proteins may be responsible for the decrease in fertility in sperm cryopreservation, such as: heat-shock protein 90 [23] and, Enolase1 (ENO1). However single protein bioresearch can only partly explain the cryodamage, and further study should be based on direct or indirect protein-protein interaction and mechanistic factors.

Proteomics technology has been identified as valuable tool for sperm [15, 24, 25]. Proteomic changes in human sperm as a result of cryoinjury have been reported previously. Wang et.al [13] found twenty-seven proteins that differed in abundance between fresh and cryopreserved sperm, by using two-dimensional polyacrylamide gel electrophoresis (2-DE) and mass spectrometry. However 2-DE has its limitations, including a low sensitivity of the densitometry analysis. Bogle et al. [26] used tandem mass tag (TMT) technology to identify potential proteomic changes at every stage of the cryopreservation process, but they did not compare fresh and cryopreserved sperm. Different from TMT, the DIA strategy has the characteristics of high quantitative accuracy and high reproducibility [11, 27]. Due to its global nature and enormous multiplexing capacity, DIA has been widely used in mechanistic studies and clinical biomarker screening in human reproduction for the enhanced protein coverage and analytical reproducibility [28]. In our study, a total of 174 significantly differential proteins were identified from 3790 quantitatively analyzed proteins, which is much more than previously reported discoveries $[13,26]$.

Improving upon previous studies on human cryopreserved sperm proteomics analysis [13, 26], our study performed KEGG analysis for the different proteins. KEGG is a frontier interdisciplinary subject based on life science and computer science. Bioinformatics analysis has been widely used as a powerful tool for data processing and prediction by employing various databases, and it has been widely used in the discovery of new biomarkers 


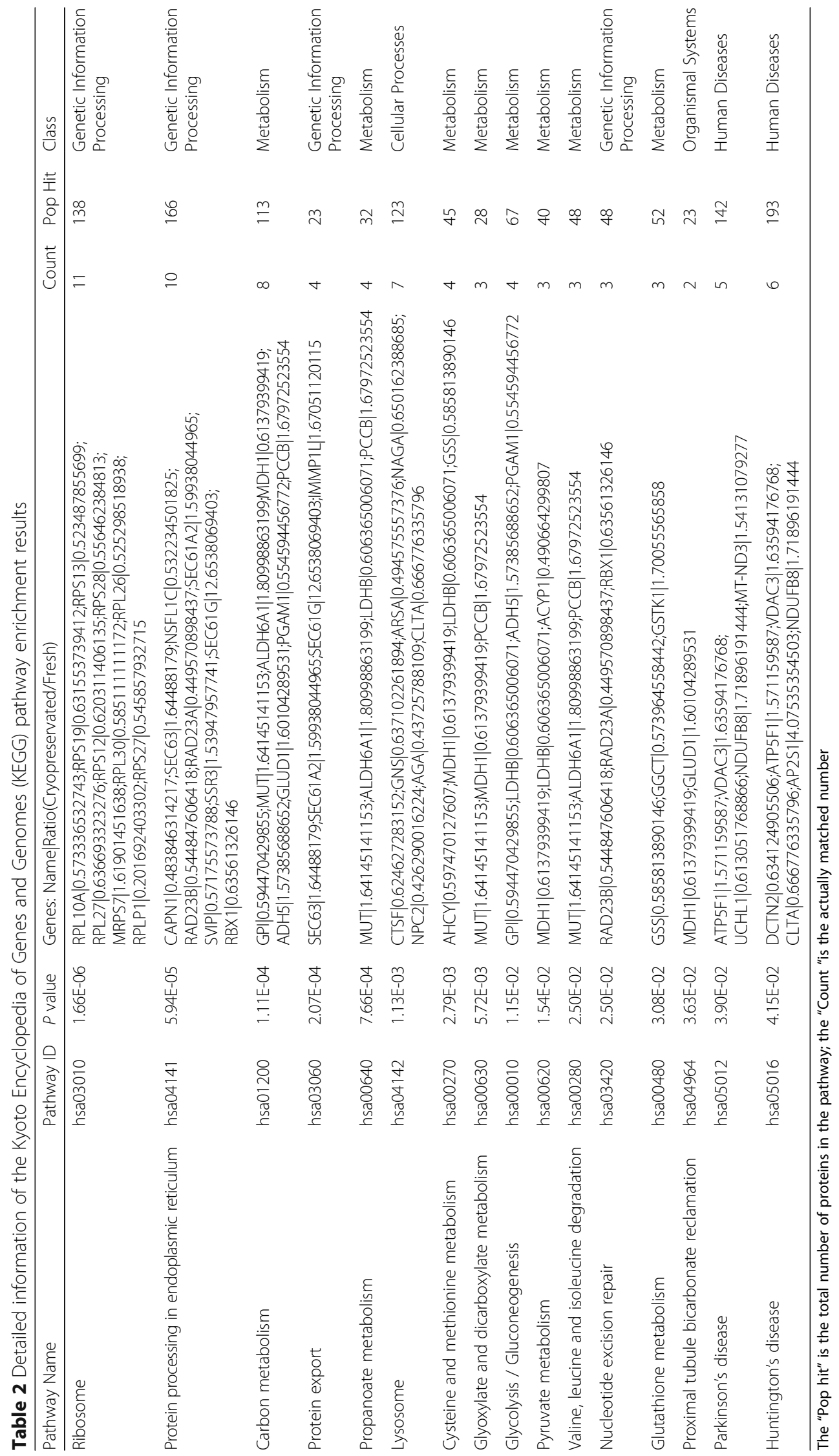



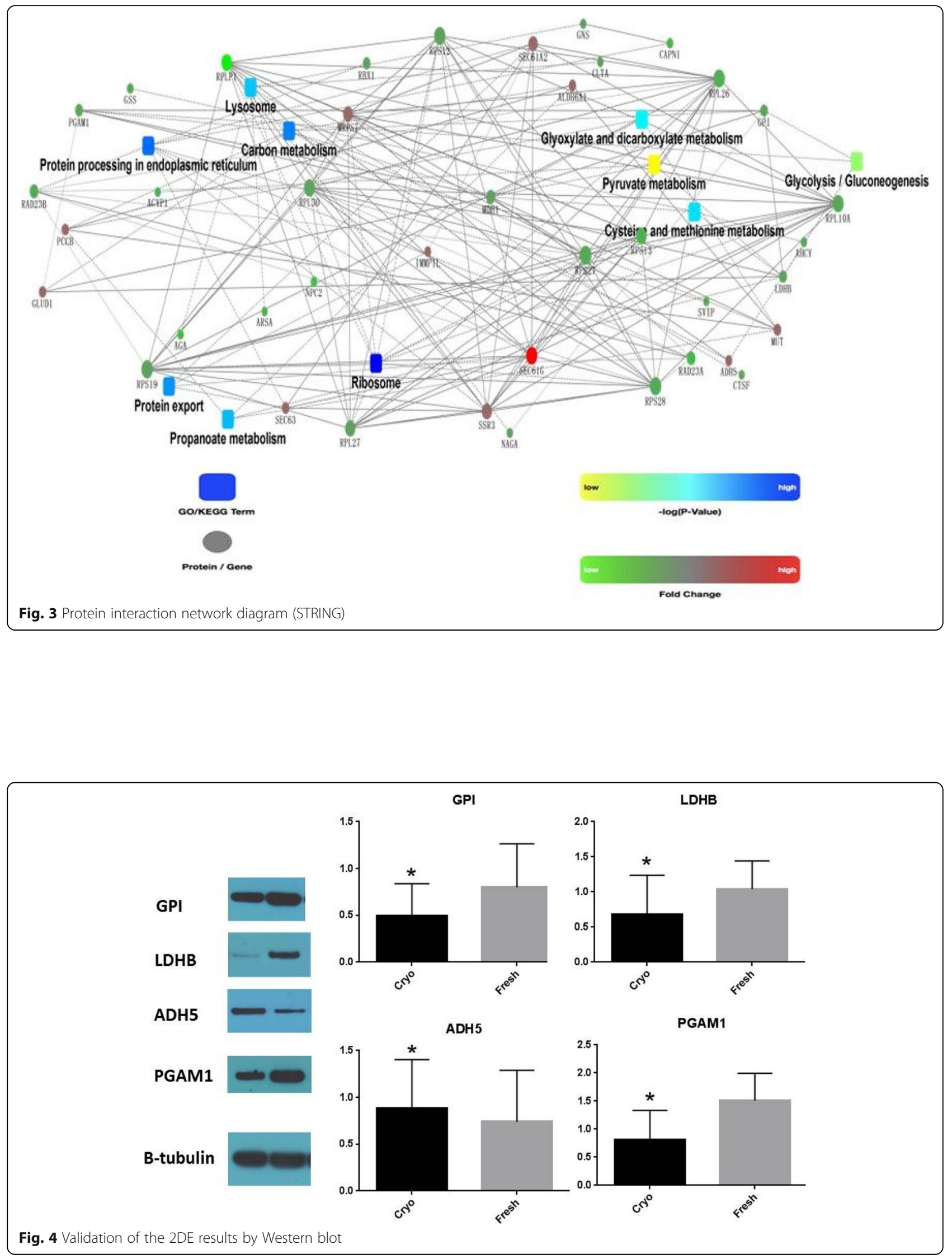
and the study of new therapeutic targets $[29,30]$. According to the KEGG analysis, the present study revealed that the metabolic pathways were affected by cryopreservation. This result confirmed previous research. Wang et al. found the decreased abundance of succinyl-CoA:3-ketoacid CoA transferase (OXCT1) in cryopreservated sperm, and this enzyme is involved in ketone metabolism and might be associated with glycolysis in sperm [13]. According to Bogle et al. [26], energy- and metabolism- related proteins account for $15 \%$ of the collated differential proteins.

Different studies have proven that the pathway of carbon metabolism is associated with sperm and male infertility [31]. In addition, sperm are highly specialized mammalian cells; the sperm must reserve enough adenosine triphosphate (ATP) to maintain the physiological processes, including motility, capacitation, hyperactivation, acrosome reaction and fertilization, all of which are highly energy dependent processes. The ATP is formed via two metabolic pathways: glycolysis and oxidative phosphorylation (OXPHOS) [32], which are extremely down regulated in the post-thaw sperm. The present study is beneficial for the further study of energy metabolic pathways involved in cryoinjury.

While many studies have reported that glycolysis is the primary source of ATP during sperm motility [32-34], OXPHOS is involved in maturation and differentiation [35]. Many study have also shown that sperm motility will significantly decrease in the process of cryopreservation and that ATP is extremely decrease in the post-thaw sperm $[5,36,37]$. Based on thede results, we focuesd the glycolysis pathway in future research. The four proteins related to glycolysis were identified by Western boltting. This study shows the a correlation between GPI and sperm motility. Consistent with previous research [37], our study show that GPI is an important factor of thawed sperm. The variability of LDHB in the process of sperm cryopreservation has also been identified in the sturgeon [38]. Early research suggests that PGAM [39] and ADH [40] is associated with spermatogenic distinction and affects the function of cell proliferation, apoptosis and migration. Further research on the correlation between PGAM1/ADH and sperm cryopreservation requiresis needed.

\section{Conclusions}

Human sperm cryopreservation is a simple and effective approach for male fertility preservation. To identify potential proteomic changes in this process, Data-independent acquisition (DIA), a proteomics technology with high quantitative accuracy and high reproducibility was used to quantitatively characterize the proteomics of human sperm cryopreservation. A total of 174 significantly differential proteins were identified between fresh and cryoperservated sperm: 98 were decreased and 76 were increased in the cryopreservation group. Bioinformatic analysis revealed that metabolic pathways play an important role in cryopreservation, including propanoate metabolism, glyoxylate and dicarboxylate metabolism, glycolysi/gluconeogenesis, and pyruvate metabolism. The four different proteins involved in glycolysis were identified by Western Blotting: GPI, LDHB, ADH5, and PGAM1. Our work will provide valuable information for future investigations and pathological studies involving sperm cryopreservation.

\section{Supplementary information}

Supplementary information accompanies this paper at https://doi.org/10. 1186/s12894-019-0565-2.

Additional file 1: Table S1. The differential proteins due to cryopreservtaion.

Additional file 2: Table S2. The results of the GO enrichment.

\section{Abbreviations}

BP: Biological process; CC: Cellular component; DDA: Data-dependent acquisition; DIA: Data-independent acquisition; FWHM: Full-Width HalfMaximum; MF: Molecular function; PPI: Protein-protein interaction; VAP: Average path velocity; VCL: Curvilinear velocity; VSL: Straight line velocity

Acknowledgements

The authors wish to thank PhD. Guibin Wang, Beijing Proteome Research Center, forthe helpful assistance in DIA.

\section{Authors' contributions}

LLF and YQG conceived and supervised the study design. LLF, QA, KSZ and $Y L$ established the mass spectrometry workflow and performed the proteomic analysis. YT, XWW, JFX, FZ and YG analyzed proteomic data and performed bioinformatics analysis. WHL and XWL verify and interpretation all the data. LLF and YL drafted the manuscript. YQG, WHL, and XWL revised the manuscript. All authors have read and approved the manuscript.

\section{Funding}

This work was supported by the CAMS Innovation Fund for Medical Sciences [2018-12M-1-004], the Chinese Medical Association clinical medical research specific fund [18010310760], the National "13th Five-Year" Plan for Science and Technology Support [2018YFC1003601], Fundamental Research Funds for the Central Universities [3332018187], National Natural Science Foundation of China [81803116], and Suzhou Science \& Technology Planning Project [sys2018077].

2018-I2M-1-004, 18010310760 and 2018 YFC1003601 support the design of the study. 3332018187, 81803116 and sys2018077 were used for purchasing most of the experimental reagents, and materials.

\section{Availability of data and materials}

The datasets for the current study are available from the corresponding author upon reasonable request.

\section{Ethics approval and consent to participate}

Experimental procedures were performed in accordance with the Ethical Principles in Human Research. The study was approved by National Research Institute for Family Planning Ethics Committee on human subjects (2018018). And written informed consent was obtained from all the sperm donors involved in the study.

\section{Consent for publication}

Not applicable

Competing interests

None 


\section{Author details}

${ }^{1}$ National Health Commission Key Laboratory of Male Reproductive Health, National Research Institute for Family Planning, Beijing 100081, China. ${ }^{2}$ Department of Male Clinical Research/Human sperm bank, National Research Institute for Family Planning \& WHO Collaborating Center for Research in Human Reproduction, Beijing 100081, China. ${ }^{3}$ Graduate School of Peking Union Medical College, Beijing 100730, China. ${ }^{4}$ Department of Reproductive Medicine, The Afliated Hospital of Qingdao University, Qingdao, Shandong 266000, People's Republic of China. ${ }^{5}$ Institute of Pediatric Research, Children's Hospital of Soochow University, Suzhou, Jiangsu Province 215025, China.

Received: 9 September 2018 Accepted: 2 December 2019 Published online: 16 December 2019

\section{References}

1. Yogev L, Kleiman SE, Shabtai E, Botchan A, Paz G, Hauser R, Lehavi O, Yavetz H, Gamzu R. Long-term cryostorage of sperm in a human sperm bank does not damage progressive motility concentration. Hum Reprod. 2010;25(5):1097-103.

2. Tournaye $H$, Dohle GR, Barratt CL. Fertility preservation in men with cancer. Lancet. 2014;384(9950):1295-301.

3. Oktay K, Harvey BE, Partridge AH, Quinn GP, Reinecke J, Taylor HS, Wallace WH Wang ET, Loren AW. Fertility Preservation in Patients With Cancer: ASCO Clinical Practice Guideline Update. J Clin Oncol. 2018;36(19):1994-2001.

4. Fu L, Zhang K, An Q, Zhou F, Lu W, Liang X, Gu Y. Low utilization of fertility preservation among Chinese male cancer patients. Int J Clin Exp Med. 2018; 11(9):9916-20.

5. Raad G, Lteif L, Lahoud R, Azoury J, Azoury J, Tanios J, Hazzouri M, Azoury J. Cryopreservation media differentially affect sperm motility, morphology and DNA integrity. Andrology. 2018;6(6):836-45.

6. Gomez-Torres MJ, Medrano L, Romero A, Fernandez-Colom PJ, Aizpurua J. Effectiveness of human spermatozoa biomarkers as indicators of structural damage during cryopreservation. Cryobiology. 2017;78:90-4.

7. Ribas-Maynou J, Fernandez-Encinas A, Garcia-Peiro A, Prada E, Abad C, Amengual MJ, Navarro J, Benet J. Human semen cryopreservation: a sperm DNA fragmentation study with alkaline and neutral comet assay. Andrology. 2014:2(1):83-7.

8. Kathrins M, Abhyankar N, Shoshany O, Liebermann J, Uhler M, Prins G, Niederberger C. Post-thaw recovery of rare or very low concentrations of cryopreserved human sperm. Fertil Steril. 2017;107(6):1300-4.

9. De Rose MB, Piccolomini MM, Belo ASS, Borges E, Jr., Filho FF: Proteomics in Human Reproduction. Protein and peptide letters 2018.

10. Cao X, Cui Y, Zhang X, Lou J, Zhou J, Bei H, Wei R. Proteomic profile of human spermatozoa in healthy and asthenozoospermic individuals. Reprod Biol Endocrinol. 2018;16(1):16.

11. Tsou CC, Avtonomov D, Larsen B, Tucholska M, Choi H, Gingras AC, Nesvizhskii Al. DIA-Umpire: comprehensive computational framework for data-independent acquisition proteomics. Nat Methods. 2015;12(3):258-64 $257 \mathrm{p}$ following 264

12. Yang X, Guo Y, Cao XF, Jia YF, Wang XW, Xu JF, Zhou F, Li H, Liang XW, Lu $W H$, et al. Effect of cryopreservation on DNA methylation status of imprited genes in human sperm. J Reprod Medcine. 2015;24(5):402-8.

13. Wang S, Wang W, Xu Y, Tang M, Fang J, Sun H, Sun Y, Gu M, Liu Z, Zhang $Z$, et al. Proteomic characteristics of human sperm cryopreservation. Proteomics. 2014;14(2-3):298-310.

14. Hezavehei M, Sharafi M, Kouchesfahani HM, Henkel R, Agarwal A, Esmaeili V, Shahverdi A. Sperm cryopreservation: a review on current molecular cryobiology and advanced approaches. Reprod BioMed Online. 2018;37(3):327-39.

15. Wang G, Wu Y, Zhou T, Guo Y, Zheng B, Wang J, Bi Y, Liu F, Zhou Z, Guo X, et al. Mapping of the N-linked glycoproteome of human spermatozoa. J Proteome Res. 2013;12(12):5750-9.

16. Li F, Zhao D, Yang S, Wang J, Liu Q, Jin X, Wang W. ITRAQ-based proteomics analysis of Triptolide on human A549 lung adenocarcinoma cells. Cell Physiol Biochem. 2018;45(3):917-34.

17. Kanehisa M, Furumichi M, Tanabe M, Sato Y, Morishima K. KEGG: new perspectives on genomes, pathways, diseases and drugs. Nucleic Acids Res. 2017;45(D1):D353-d361.

18. Szklarczyk D, Franceschini A, Wyder S, Forslund K, Heller D, Huerta-Cepas J, Simonovic M, Roth A, Santos A, Tsafou KP, et al. STRING v10: protein-protein interaction networks, integrated over the tree of life. Nucleic Acids Res. 2015:43(Database issue):D447-52.
19. Liu Y, Wang J, Qin Y, Huang C, Archacki S, Ma J, Li D, Liu M. Identification of three mutations in the MVK gene in six patients associated with disseminated superficial actinic porokeratosis. Clinica Chimica Acta; Int J Clin Chem. 2016:454:124-9.

20. Agarwal A, Mulgund A, Hamada A, Chyatte MR. A unique view on male infertility around the globe. Reprod Biol Endocrinol. 2015;13:37.

21. Nahata L, Caltabellotta NM, Yeager ND, Lehmann V, Whiteside SL, O'Brien SH, Quinn GP, Gerhardt CA: Fertility perspectives and priorities among male adolescents and young adults in cancer survivorship. Pediatric blood \& cancer 2018.

22. Isaac AV, Kumari S, Nair R, Urs DR, Salian SR, Kalthur G, Adiga SK, Manikkath J, Mutalik S, Sachdev D, et al. Supplementing zinc oxide nanoparticles to cryopreservation medium minimizes the freeze-thaw-induced damage to spermatozoa. Biochem Biophys Res Commun. 2017;494(3-4):656-62.

23. Cao WL, Wang YX, Xiang ZQ, Li Z. Cryopreservation-induced decrease in heat-shock protein 90 in human spermatozoa and its mechanism. Asian J Androl. 2003;5(1):43-6.

24. Li CJ, Wang D, Zhou X. Sperm proteome and reproductive technologies in mammals. Anim Reprod Sci. 2016:173:1-7.

25. Kosteria I, Anagnostopoulos AK, Kanaka-Gantenbein C, Chrousos GP, Tsangaris GT. The Use of Proteomics in Assisted Reproduction. In Vivo (Athens, Greece). 2017:31(3):267-83.

26. Bogle OA, Kumar K, Attardo-Parrinello C, Lewis SE, Estanyol JM, Ballesca JL, Oliva R. Identification of protein changes in human spermatozoa throughout the cryopreservation process. Andrology. 2017;5(1):10-22.

27. Muntel J, Xuan Y, Berger ST, Reiter L, Bachur R, Kentsis A, Steen H. Advancing urinary protein biomarker discovery by data-independent acquisition on a Quadrupole-Orbitrap mass spectrometer. J Proteome Res. 2015;14(11):4752-62

28. Meyer JG, D'Souza AK, Sorensen DJ, Rardin MJ, Wolfe AJ, Gibson BW, Schilling B. Quantification of lysine acetylation and Succinylation stoichiometry in proteins using mass spectrometric data-independent acquisitions (SWATH). J Am Soc Mass Spectrom. 2016;27(11):1758-71.

29. Chen L, Lu D, Sun K, Xu Y, Hu P, Li X, Xu F. Identification of biomarkers associated with diagnosis and prognosis of colorectal cancer patients based on integrated bioinformatics analysis. Gene. 2019.

30. Zhang $Y$, Tang $X$, Pang Y, Huang L, Wang D, Yuan C, Hu X, Qu L. The Potential Mechanism of Bufadienolide-Like Chemicals on Breast Cancer via Bioinformatics Analysis. Cancers (Basel). 2019;11(1):91.

31. Singh K, Jaiswal D. One-carbon metabolism, spermatogenesis, and male infertility. Reprod Sci (Thousand Oaks, Calif). 2013;20(6):622-30.

32. du Plessis SS, Agarwal A, Mohanty G, van der Linde M. Oxidative phosphorylation versus glycolysis: what fuel do spermatozoa use? Asian J Androl. 2015;17(2):230-5.

33. Gibb Z, Aitken RJ. The impact of sperm metabolism during in vitro storage: the stallion as a model. Biomed Res Int. 2016;2016:9380609.

34. Hereng TH, Elgstoen KB, Cederkvist FH, Eide L, Jahnsen T, Skalhegg BS, Rosendal KR. Exogenous pyruvate accelerates glycolysis and promotes capacitation in human spermatozoa. Hum Reprod. 2011;26(12):3249-63.

35. Stendardi A, Focarelli R, Piomboni P, Palumberi D, Serafini F, Ferramosca A, Zara V. Evaluation of mitochondrial respiratory efficiency during in vitro capacitation of human spermatozoa. Int J Androl. 2011;34(3):247-55.

36. O'Connell M, McClure N, Lewis SE. The effects of cryopreservation on sperm morphology, motility and mitochondrial function. Hum Reprod. 2002;17(3):704-9.

37. Jiang XP, Wang SQ, Wang W, Xu Y, Xu Z, Tang JY, Sun HY, Wang ZJ, Zhang W. Enolase1 (ENO1) and glucose-6-phosphate isomerase (GPI) are good markers to predict human sperm freezability. Cryobiology. 2015;71(1):141-5.

38. Li P, Guo W, Yue H, Li C, Du H, Qiao X, Liu Z, Zhou Q, Wei Q. Variability in the protein profiles in spermatozoa of two sturgeon species. PLoS One. 2017;12(10):e0186003.

39. Zhang $S$, Zhao Y, Lei B, Li C, Mao X. PGAM1 is Involved in Spermatogenic Dysfunction and Affects Cell Proliferation, Apoptosis, and Migration. Reprod Sci (Thousand Oaks, Calif). 2015;22(10):1236-42.

40. Aly HA, El-Beshbishy HA, Banjar ZM. Mitochondrial dysfunction induced impairment of spermatogenesis in LPS-treated rats: modulatory role of lycopene. Eur J Pharmacol. 2012;677(1-3):31-8.

\section{Publisher's Note}

Springer Nature remains neutral with regard to jurisdictional claims in published maps and institutional affiliations. 